\title{
A Rare Manifestation of Crohn's Disease: Sinonasal Granulomatosis. Report of a Case and Review of Literature
}

\begin{abstract}
Lilia Baili, Imène Rachdi, Fatma Daoud, Zohra Aydi, Besma Ben Dhaou, Samir Kochbati, Fatma Boussema Department of Internal Medicine, Habib Thameur Hospital, University El Manar, Tunis
\end{abstract}

\begin{abstract}
:
Crohn's disease is a granulomatous inflammatory bowel disease. Its pathologic findings include non-contiguous chronic inflammation and non-caseating granulomas, sometimes with extra-intestinal localizations. Sinonasal manifestations of Crohn's disease are quite rare and only a few cases have been reported up to date in the worldwide literature. They are characterized by chronic mucosal inflammation, obstruction, bleeding and occasionally septal perforation. We report a case of sinonasal granulomatosis revealing Crohn's disease in a 22-year-old woman and go over the available literature on sinonasal involvement in Crohn's disease.
\end{abstract}

Keywords: Crohn's disease, granulomatosis, nasal involvement, sinusitis, ulcerative colitis, inflammatory bowel disease

Received: $30 / 07 / 2014$

Accepted: 03/09/2014

Published: 01/10/2014

How to cite this article: Baili L, Rachdi I, Daoud F, Aydi Z, Ben Dhaou B, Kochbati S, Boussema F. A Rare Manifestation of Crohn's Disease:

Sinonasal Granulomatosis. Report of a Case and Review of Literature, EJCRIM 2014;1:doi: 10.12890/2014_000123

Conflicts of Interests: The authors declare that they have no conflicts of interest related to this research. 


\section{Introduction}

Crohn's disease (CD) is an inflammatory bowel disease of unknown aetiology characterized by chronic relapsing and remitting granulomatous inflammation of the alimentary tract [1]. CD involves the distal ileum and the colon most often [1]. The extra-intestinal manifestations of $C D$, especially involvement of the head and neck, are uncommon and, in rare circumstances, may dominate the initial clinical presentation. Nasal localizations are rare and are characterized by chronic mucosal inflammation, obstruction, bleeding and occasionally septal perforations [2]. Nasal endoscopic findings are non-specific and can be caused by many diseases such as tuberculosis, syphilis, sarcoidosis, Wegener's granulomatosis and angioedema. We report a case of sinonasal mucosa involvement in a 22-year-old patient revealing $C D$ and we review the available literature on sinonasal involvement in CD.

\section{Case report}

A 22-year-old woman, with no medical history, was admitted for nasal obstruction and recurrent epistaxis that had started 5 months earlier. She complained also of mucosanguineous diarrhoea, weight loss and abdominal cramps. She did not have fever, genital ulcers, arthralgia or ocular symptoms. Her familial history was unremarkable, especially with regard to angioedema and chronic inflammatory bowel diseases. On admission, she was cachectic with a weight of $38 \mathrm{~kg}$

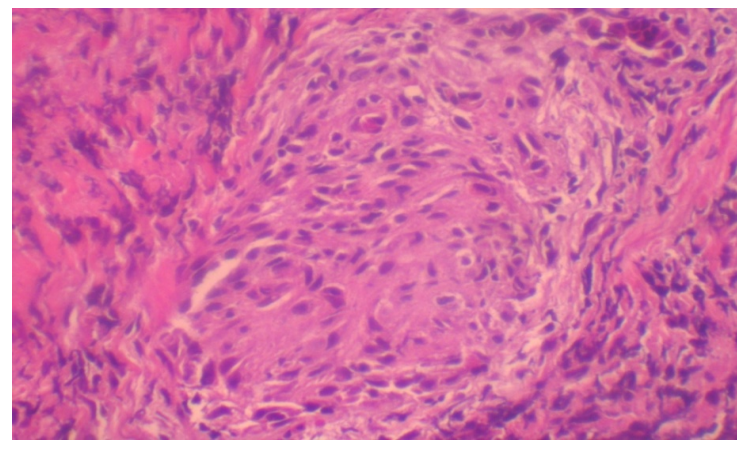

Figure 1: Histopathological examination of nasal biopsy (haematoxylin and eosin staining): non-caseating granuloma, epithelioid and multinuclear cells.

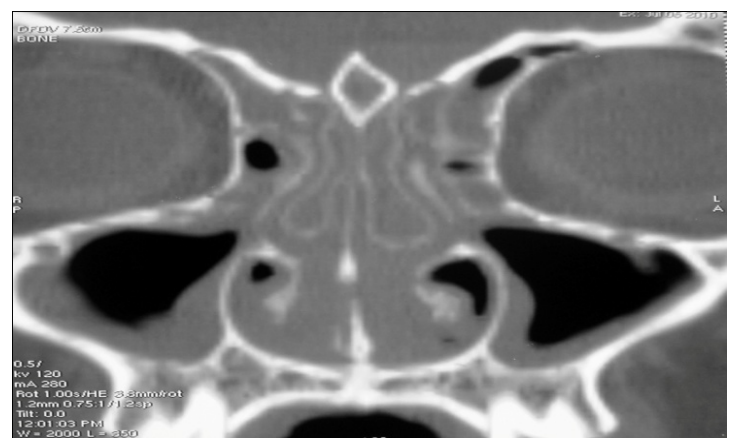

Figure 2: Unenhanced computed tomography of paranasal sinuses: complete opacification of the ethmoid sinuses and mucosal thickening of the and a body mass index of $16 \mathrm{~kg} / \mathrm{m}^{2}$. Her temperature was $37.8^{\circ} \mathrm{C}$, and she had an alteration of general status and tenderness of the right iliac fossa. There were no lymph node, hepatosplenomegaly or skin lesions. Digital rectal examination showed blood and mucus in the stool. There was no proteinuria or haematuria on urinary bands. Laboratory investigations revealed normocytic anaemia, with a haemoglobin level of $9.8 \mathrm{mg} / \mathrm{dl}$, and normal white blood cell count and leukocyte formula. Biological inflammatory syndrome was noted (erythrocyte sedimentation rate $45 \mathrm{~mm} / \mathrm{h}$, C-reactive protein $96 \mathrm{mg} / \mathrm{l})$. Biological signs of malabsorption were present, with cholesterol level at 2.1 $\mathrm{mmol} / \mathrm{l}(3.6-5.2 \mathrm{mmol} / \mathrm{l})$, low level of ferritinaemia at $8 \mathrm{ng} / \mathrm{ml}$ (14-135 $\mathrm{ng} / \mathrm{ml}$ ), hypoprotidaemia and hypoalbuminia at $22 \mathrm{~g} / \mathrm{l}(40-47 \mathrm{~g} / \mathrm{l})$. Hepatic tests, levels of lactate dehydrogenase and uric acid were normal. Chest X-ray and abdominal ultrasound were normal. The anterior rhinoscopy exhibited nasal septal deviation and easy mucosal bleeding. A nasal septum mucosa biopsy was performed. Pathologic examination of this specimen revealed non-necrotizing granulomas with no vasculitis signs or eosinophilic infiltration (Fig. 1).

No foreign bodies, fungi or acid-fast bacilli were identified. A computed tomography scan of the sinus showed pansinusitis and diffuse thickening of the entire sinonasal mucosa, particularly of the septum (Fig. 2). 


\section{European Journal \\ of Case Reports in \\ Internal Medicine}

Salivary gland biopsy showed granulomas. The diagnosis of sinonasal and buccal granulomatosis was retained. Based on patient anamnesis, toxic and drug-induced granulomatosis were improbable.

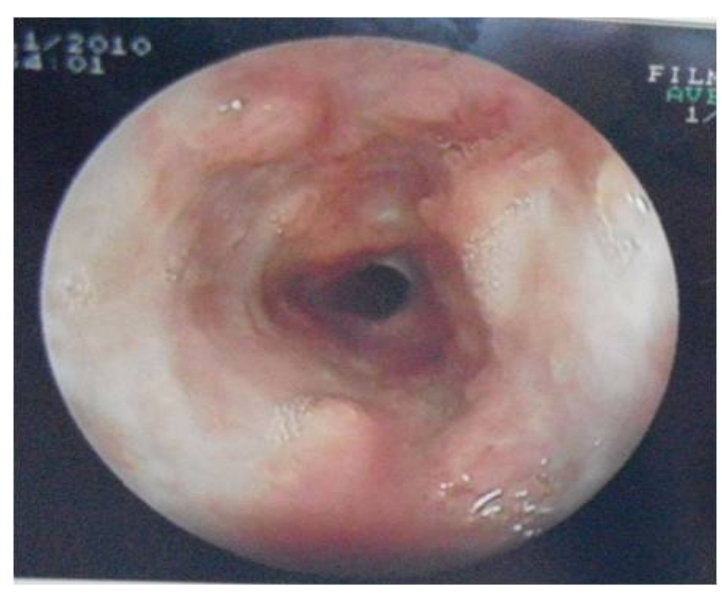

Figure 3: Oesophago-gastro-duodenoscopy examination showed oesophageal stenosis with pseudopolypoid formation.

To differentiate infectious diseases that lead to nasal granulomatous, brucellosis, typhoid fever, syphilis, viral hepatitis B and C, cytomegalovirus, Epstein-Barr virus and HIV were studied, yielding negative results for all tests. Tuberculin reaction and sputum acid-fast bacilli were negative.

Systemic vasculitis, such as Wegener's disease and Churg-Strauss syndrome, and microscopic polyangiitis were improbable because of the absence of renal, pulmonary and neurological involvement and negative anti-neutrophil cytoplasmic antibodies. A sarcoidosis was improbable because of the absence of mediastino-pulmonary involvement and a normal angiotensin-converting enzyme (ACE) level. Based on iron-deficiency anaemia and mucosanguineous diarrhoea, oesophago-gastro-duodenoscopy was performed, revealing oesophageal stenosis with pseudopolypoid formation (Fig. 3).

Histopathological examination of the biopsy of this area revealed severe oesophagitis.

Colonoscopy showed a red, swollen mucosa with multiple erosive lesions of the whole intestine with an acute ileitis and aphthous ulcerations (Fig. 4). Histopathological examination of colic biopsies showed focal patchy acute inflammation and focal crypt architectural irregularity, which was consistent with CD (Fig. 5).

Diagnosis of sinonasal and buccal granulomatosis as the first manifestation of CD with multiple digestive involvements was retained. Steroid therapy with prednisone was prescribed at the dosage of $35 \mathrm{mg}$ once a day for 4 weeks with resolution of nasal obstruction and diarrhoea. While degression of corticosteroids, she presented a recurrence of diarrhoea. An immunosuppressant (azathioprine: $100 \mathrm{mg} /$ day) was then started. The patient was asymptomatic and the nasal mucosa had a normal appearance during the 2 years of follow-up.

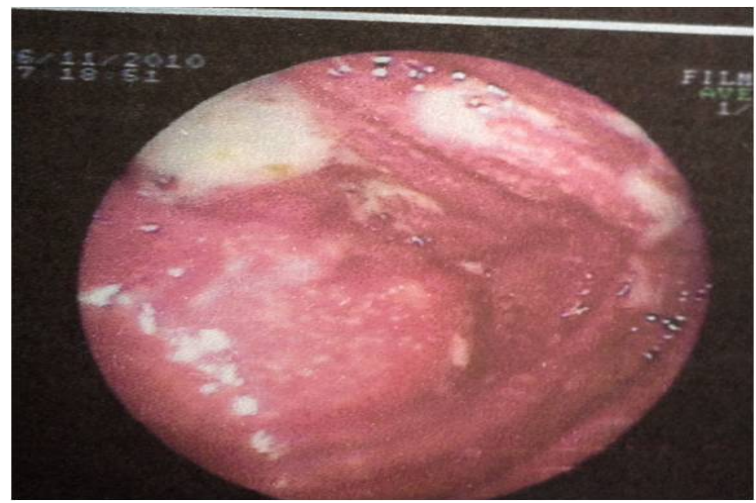

Figure 4: Oesophago-gastro-duodenoscopy examination showed oesophageal stenosis with pseudopolypoid formation.

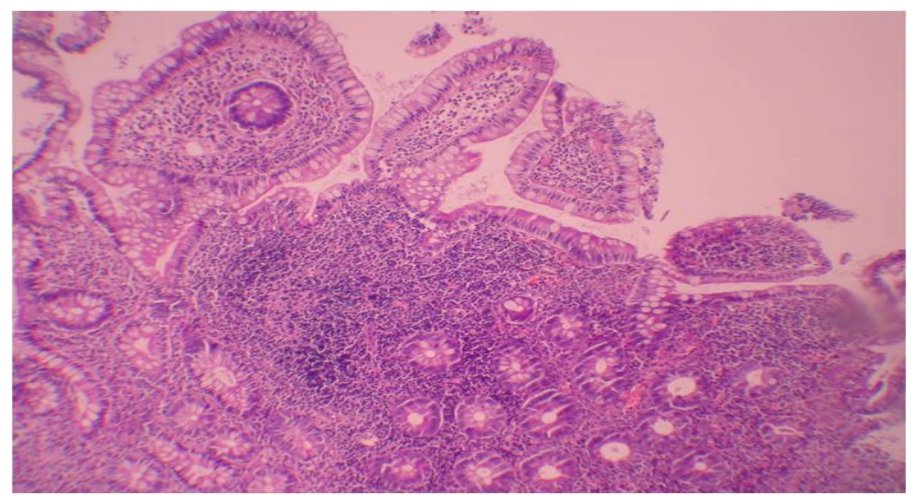

Figure 5: Histopathological examination of colic biopsy (haematoxylin and eosin staining): patchy acute inflammation and focal crypt irregularity. 


\section{Discussion}

In a series of 700 patients with CD reviewed by Greenstein et al. [3], 36\% had extra-intestinal manifestations. Otolaryngologic involvement is more common in men and in the younger population [4]. Extra-intestinal manifestations may present at any time during the disease process and can even be the initial presentation of the disease [5]. The mucocutaneous manifestations of $C D$ in the oro-facial region are multiple [6]. Oral ulcers of the tongue, buccal mucosa and palate are the most frequent lesions [2-4, 7]. Very few reports of laryngeal CD exist in the literature [7-9]. Nasal manifestations are extremely rare [10-12].

Our case is a new case of authentic sinonasal granulomatosis that revealed CD. The sinonasal complaints may be nasal obstruction, epistaxis, impairment of smell, purulent rhinorrhoea crusting, acute or chronic sinusitis and deformity of the nasal pyramid. In our patient, nasal obstruction and recurrent epistaxis were the main symptoms. Chronic atrophic or scabby rhinitis, oedema, ulcerations and polyposis of nasal mucosa, perforation of nasal septum, lysis and necrosis of the turbinates, saddle nose deformity, stenosis of the nasal fossae and empty nose syndrome were the most common endoscopic findings in these patients [10-13]. Nasal involvement with septum perforation, excoriation of the left nasal septum and abundant mucus revealed CD in only one patient [14]. Histopathologic examination of nasal biopsies may demonstrate non-specific chronic inflammation [15]. It also showed non-caseating granulomas in the majority of patients, as in our case [10-13]. Pharyngeal or laryngeal involvement, pansinusitis, peritonsillitis and oral ulcerations were also described in some of these patients [12]. Although the presence of granulomas is helpful in establishing the diagnosis of $C D$, they are not pathognomonic and other granulomatous processes and infectious aetiologies must be ruled out. Our patient did not have any history of drug use, nasal trauma or surgery. Inflammatory disorders and infections were excluded by appropriate laboratory tests. Classically, sinonasal manifestations are considered 'a relapse of the disease', so they justify an adjustment of medical treatment [15]. Severe cases of intestinal CD have been treated with 5-aminosalicylic acid products with or without steroids. In extra-intestinal sites, topical steroids can be used first and have been shown to be successful in up to $50 \%$ of oral ulcers [4]. If these fail, oral corticosteroids become the mainstay of therapy. However, due to significant morbidity associated to this treatment and to cases that are refractory, azathioprine and methotrexate have been used. Unfortunately, a significant percentage of patients do not respond to azathioprine and the therapeutic efficacy of methotrexate appears to decrease with prolonged use [16]. Recently, infliximab, a chimeric anti-tumour necrosis factor alpha antibody, has shown promise in the management of CD [16]. The mucosa in CD patients has a higher concentration of the cytokine tumour necrosis factor- $\alpha$. Infliximab blocks this potent proinflammatory cytokine. The only reports of infliximab use for the extra-intestinal manifestations of $C D$ are arthritis, which responds well.

\section{Learning Points}

- Extra-intestinal manifestations of CD may appear at any time during the course of the disease process and may be the initial symptom.

- Endoscopic nasal findings are non-specific and may be confused with many other diseases.

- Awareness of sinonasal manifestations will prevent misdiagnosis or a delay in diagnosis.

- Corticosteroid therapy is the current mainstay of therapy.

- Infliximab may be useful for the treatment of extra-intestinal manifestations of CD. 


\section{European Journal \\ of Case Reports in \\ Internal Medicine}

\section{References}

1. Mamula P, Markowitz JE, Baldassano RN. Inflammatory bowel disease in early childhood and adolescence: special considerations, Gastroenterol Clin North Am 2003;32:967-995.

2. Kriskovich MD, Kelly SM, Jackson WD. Nasal septal perforation: a rare extraintestinal manifestation of Crohn's disease, Ear Nose Throat J 2000;79:520-523.

3. Greenstein AJ, Janowitz HD, Sachar DB. The extra-intestinal complications of Crohn's disease and ulcerative colitis: a study of 700 patients, Medicine (Baltimore) 1976;55:401-412.

4. Plauth M, Jenss H, Meyle J. Oral manifestations of Crohn's disease: an analysis of 79 cases, J Clin Gastroenterol 1991;13:29-37.

5. Dupuy A, Cosnes J, Revuz J, Delchier JC, Gendre JP, Cosnes A. Oral Crohn's disease: clinical characteristics and long-term follow-up of 9 cases, Arch Dermatol 1999;135:439-442.

6. Fatahzadeh M, Schwartz RA, Kapila R, Rochford C. Orofacial Crohn's disease: an oral enigma, Acta Dermatovenerol Croat 2009;17:289-300.

7. Croft CB, Wilkinson AR. Ulceration of the mouth, pharynx, and larynx in Crohn's disease of the intestine, $B r J$ Surg 1972;59:249-252.

8. Bishop RP, Brewster AC, Antonioli DA. Crohn's disease of the mouth, Gastroenterology 1972;62:302-306.

9. Wilder WM, Slagle GW, Hand AM, Watkins WJ. Crohn's disease of the epiglottis, aryepiglottic folds, anus, and rectum, J Clin Gastroenterol 1980;2:87-91.

10. Sari S, Dalgic B, Yilmaz M, Poyraz A. Nasal septal perforation in an adolescent girl with Crohn's disease: a rare extraintestinal manifestation, Dig Dis Sci 2007;52:1285-1287.

11. Grasso DL, Bussani M, Martelossi S. Choanal stenosis in Crohn's disease: a case report and review of literature, Int J Pediatr Otorhinolaryngol 2007;2:228-230.

12. Pellicano R, Sostegni R, Sguazzini C, Reggiani S, Astegiano M. A rare location of Crohn's disease: the nasal mucosa, Acta Biomed 2011;82:74-76.

13. Oghan F, Pekkan G, Ozveren O. Saddle nose deformity, palatal perforation and truncus arteriosus in a patient with Crohn's disease, J Craniomaxillofac Surg 2012;40:17-19.

14. Kryssia RC, Henry ZB. Nasal septum perforation: rare manifestation of Crohn's disease, Indian J Gastroenterol 2006;25:214.

15. Eloy $P$, Leruth $E$, Goffart $Y$, Nollevaux $M C$, Fridman $V$, Dartevelle $M$ et al. Sinonasal involvement as a rare extraintestinal manifestation of Crohn's disease, Eur Arch Otorhinolaryngol 2007;264:103-108.

16. Van Deventer SJ. Anti-TNF antibody treatment of Crohn's disease, Ann Rheum Dis 1999;58:114-120. 